\title{
SS-Coprime Modules
}

Inaam M.A.Hadi

Department of Mathematics, College of Education for Pure Science,

\section{Abstract}

University of Baghdad

Let $\mathrm{R}$ be a commutative ring with unity and $\mathrm{M}$ is a unitary left $\mathrm{R}$-module . In this paper, we introduce the notion of strongly S-coprime modules, where $M$ is called strongly S-coprime briefly (SS-Coprime) if for each $r \in R, r^{2} M$ is small in $M$ implies $\mathrm{rM}=0$. We investigate many properties about this concept. Moreover many relations between it and other related concepts, are given.

Keywords: Coprime module ; S-Coprime module; SS-Coprime module; SSS-Coprime module; $\tau$-noncosingular module.

\section{Council for Innovative Research}

Peer Review Research Publishing System

Journal: JOURNAL OF ADVANCES IN MATHEMATICS

Vol.11, No. 5

www.cirjam.com, editorjam@gmail.com 


\section{INTRODUCTION}

Let $\mathrm{R}$ be a commutative ring with unity and let $\mathrm{M}$ be a unital $\mathrm{R}$-module. Recall that a proper submodule $\mathrm{N}$ of $\mathrm{M}$ is called prime if whenever $\mathrm{r} \in \mathrm{R}, \mathrm{x} \in \mathrm{M}, \mathrm{rx} \in \mathrm{N}$ implies that $x \in N$ or $r \in[N: M],[10] . \mathrm{M}$ is called a prime R-module if the zero submodule (0) is a prime submodule of $M$. Equivalently, $M$ is a prime R-module if $a n n_{R} M=a n n_{R} N$ for each nonzero submodule $\mathrm{N}$ of $\mathrm{M},[4]$.

S. Yassemi in [13] introduced the notions of second submodule and second module, where a submodule $N$ of $M$ is called second if whenever $r \in R-\{0\}, r N=N$ or $r N=0$. M is called a second module if $M$ is a second submodule of $M$; that is whenever $r \in R-\{0\}, r M=M$ or $r M=0$.

S. Annine in [2] introduced the notion of coprime module as follows: $M$ is called a coprime R-module if annM=ann $\frac{M}{N}$ for each proper submodule $\mathrm{N}$ of $\mathrm{M}$. However, it is known that second modules and coprime modules are equivalent.

Moreover I. E. Wijayant in [15], give the following :

An R-module $\mathrm{M}$ is coprime if and only if $a n n_{R} \mathrm{M}=a n n_{R} \frac{M}{N}$ for each proper fully invariant summodule $\mathrm{N}$ of $\mathrm{M}$. and proved that it is equivalent to definition of coprime module (in sense of $S$. Annine

Recall that a proper submodule $\mathrm{N}$ of $\mathrm{M}$ is called small (denoted by $\mathrm{N}<<\mathrm{M}$ ) if whenever $\mathrm{N}+\mathrm{K}=\mathrm{M}, \mathrm{K}$ is a submodule of $\mathrm{M}$, then $\mathrm{K}=\mathrm{M}$ [1]. As a generalization of coprime module. I.M.A.Hadi \& R.I.Khalaf in [7] introduce the notion of small coprime (briefly, S-coprime) module, where an R-module $\mathrm{M}$ is called S-coprime if $a n n_{R} M=a n n_{R} \frac{M}{N}$ for each $\mathrm{N}<<\mathrm{M}$. Equivalently, M is $\mathrm{S}$-coprime if for each $r \in \mathrm{R}-\{0\}, \mathrm{rM}<<\mathrm{M}$ implies $\mathrm{rM}=0$.

Tütuncu, Tribak in [12] introduced an studied the notion of $\mathrm{T}$. non cosingular, where an R-module $\mathrm{M}$ is called $\mathrm{T}$ noncosingular if whenever $\varphi \in E n d M, \operatorname{Im} \varphi<<\mathrm{M}$, implies $\operatorname{Im} \varphi=0$. It is clear that T-noncosingular module is $\mathrm{S}$-coprime and the converse is not true in general, as we shall see later.

In section two of this paper we investigate the notion of strongly S-coprime module (briefly SS-coprime) where an Rmodule $M$ is called SS-coprime if for any $a, b \in R$, $a b M<<M$ implies $a M=0$ or $b M=0$, it is clear that SS-coprime module is $s$ coprime but the converse is not true. However we see by examples that the concept SS-coprime and T-noncosingular are independent. However, if $\mathrm{M}$ is $\mathrm{T}$-noncosingular and annM is a prime ideal then $\mathrm{M}$ is SS-coprime. Moreover many properties of SS-coprime modules and some connections between SS-coprime modules and other related concepts are given.

In section three, the concept of semistrongly S-coprime (briefly, SSS-coprime) is presented, where an R-module is SSScoprime if whenever $r \in R$ with $r^{2} M<<M$, then $r M=0$. It is clear that every SS-coprime is SSS-coprime, but the converse is true under certain condition. Most of properties of SS-coprime modules generalized to SSS-coprime.

\section{SS-Coprime Modules}

\section{Definition 2.1 :}

An R-module is called strongly S-coprime (briefly SS-coprime) if for each a,beR, abM $<<\mathrm{M}$ implies $\mathrm{aM}=0$ or $\mathrm{bM}=0$

\section{Remarks and Examples 2.2:}

1) It is clear that every SS-coprime module is S-coprime, but the converse is not true in general, for example:

The $Z$-module $Z_{6}$ is $S$-coprime since $(\overline{0})$ is the only small submodule in $Z_{6}$ and so $r Z_{6}<<Z_{6}$ implies $r Z_{6}=(\overline{0})$. But $Z_{6}$ is not SS-coprime, since $2.3 Z_{6}=(\overline{0})<<Z_{6}$ and $2 . Z_{6} \neq(\overline{0}), 3 . Z_{6} \neq(\overline{0})$.

2) Let $M$ be an R-module, then $M$ is SS-coprime if and only if $M$ is $S$-coprime and annM is a prime ideal

\section{Proof :}

It is easy

3) Every T-noncosingular module is S-coprime , but neither S-coprime nor SS-coprime implies T-noncosingular in general.

\section{Proof :}

Let $\mathrm{M}$ be a T-noncosingular R-module . Let $r \in \mathrm{R}-\{0\}, \mathrm{rM}<<\mathrm{M}$. Define $\varphi: \mathrm{M} \rightarrow \mathrm{M}$ by $\varphi(\mathrm{m})=\mathrm{rm}, \forall \mathrm{m} \in \mathrm{M}$. Then $\operatorname{Im} \varphi=r \mathrm{M}<<\mathrm{M}$ and since $\mathrm{M}$ is T-noncosingular, $\mathrm{rM}=0$. Thus $\mathrm{M}$ is $\mathrm{S}$-coprime

Now consider the following example:

Let $M$ be the $Z$ module $Z_{2^{\infty}} \oplus Z_{2}$. Then $M$ is not T-noncosingular Z-module. However we can show that $M$ is $S S$ coprime so let $\mathrm{abM}<<\mathrm{M}=Z_{2^{\infty}} \oplus Z_{2}$ then ab $Z_{2^{\infty}} \ll Z_{2^{\infty}}$ and $a b Z_{2}<<Z_{2}$. But $Z_{2^{\infty}}$ is divisible so ab $Z_{2^{\infty}}<<Z_{2^{\infty}}$ implies ab=0 and hence $a=0$ or $b=0$. Thus $a M=0$ or $b M=0$, that is $M$ is SS-coprime (and hence $M$ is $S$-coprime) 


\section{ISSN 2347-1921}

4) T-noncosingular module need not be SS-coprime, for example: Th $\mathrm{Z}$-module $\mathrm{Z}_{6}$ is not SScoprime by part (1), but it is T-noncosingular .

5) If $\mathrm{M}$ is T-noncosingular and $\operatorname{ann}_{\mathrm{R}} \mathrm{M}$ is a prime ideal, then $\mathrm{M}$ is $\mathrm{SS}$-coprime .

\section{Proof :}

Let $a, b \in R, a b M<<M$. Define $f: M \rightarrow M$ byf $(m)=a b m, \forall m \in M$. Then $I m f=a b M<<M$. But $M$ is $T$-noncosingular, so $a b M=(0)$, that is $a b \in$ annM. As annM is a prime ideal, then $a \in$ ann $M$ or $b \in$ annM ; thus $a M=(0)$ or $b M=(0)$ and $M$ is SS-coprime.

6) An R-module $M$ is SS-coprime if and only if $M$ is an SS-coprime $\bar{R}$-module, where $\bar{R}=R / a n n M$

7) Let $M, M$ ' be two isomorphic R-module. Then $M$ is SS-coprime if and only if $M$ ' is SS-coprime .

8) A ring $R$ is an SS-coprime R-module if and only if $R$ is an integral domain

\section{Proof :}

Let $R$ be an integral domain , let $a, b \in R$ with $(a)(b) R<<R$. Then $(a)(b)=0$ and so $(a)=0$ or $(b)=0$. Conversely, if $R$ is $S S$ coprime R-module, let $a, b \in R$ a. $b=0$. Then $(a)(b)<<R$ and $R$ is SS-coprime, either $(a)=0$ or $(b)=0$. Thus $a=0$ or $b=0$; that is $R$ is an integral domain.

9) Let $\mathrm{R}$ be an SS-coprime, then $\mathrm{R}$ is $\mathrm{K}$-nonsingular and the converse is not true in general, where $\mathrm{R}$ is $\mathrm{K}$-nonsingular if for each $f \in R, f \neq 0$, kerf $\Varangle_{e} R$ (ker $f$ is not essential in $\left.R\right)$.

\section{Proof :}

$R$ is SS-coprime, so part $(8), J(R)=(0)$. Thus $L(R)=Z(R)=(0)$. Hence kerf $\Varangle_{e} R$.

Also $Z_{6}$ as $Z_{6}$-module is $\mathrm{K}$-nonsingular, but it is not SS-coprime .

Recall that an R-module $M$ is called a scalar module if for each $f \in E$ ndM , there exists $r \in R$ such that $f(m)=r m$ ,$\forall \mathrm{m} \in \mathrm{M}[11]$.

\section{Proposition 2.3 :}

Let $\mathrm{M}$ be an R-module. If $\mathrm{M}$ is $\mathrm{S}$-coprime and scalar module, then $\mathrm{M}$ is $\mathrm{T}$-nonsingular module.

\section{Proof :}

Let $f \in E$ End $M$ and $I m f<<M$. Since $M$ is a scalar R-module, there exists $r \in R$ such that $f(m)=r m, \forall m \in M$. Thus $f(M)=r M<<M$ and since $M$ is S-coprime $f(M)=r M=0$.

Therefore $M$ is $T$-noncosingular.

Recall that an R-module $M$ is called a multiplication $R$-module, if for each $N \leq M$ there exists an ideal I of $R$ such that $N=I M$. Equivalently, $M$ is a multiplication R-module if for each $N \leq M, N=[N: M][3]$, where[N:M] $=\{r \in R: r M \subseteq N\}$

\section{Remark 2.4 :}

Every multiplication SS-coprime R-module $\mathrm{M}$ has (0) as the only small submodule of $\mathrm{M}$

\section{Proof :}

Let $\mathrm{N}<<\mathrm{M}$. Since $\mathrm{M}$ is SS-coprime so $\mathrm{M}$ is S-coprime . Hence annM=ann $\frac{M}{N}=[\mathrm{N}: \mathrm{M}]$.

This implies $(0)=($ annM) $M=[N: M] M=N$.

\section{Proposition 2.5 :}

Let $\mathrm{M}$ be an SS-coprime $\mathrm{R}$-module. Then $\mathrm{M}$ is a prime module if and only if $\mathrm{M}$ is a primary module.

\section{Proof :}

$(\Rightarrow)$ It is clear .

$(\Leftarrow)$ Let $r \in R, x \in M$ and $r x=0$. Since $M$ is primary, either $x=0$ or $r^{n} \in$ annM for some $n \in Z_{+}$. But $M$ is SS-coprime implies ann $M$ is a prime ideal, hence either $x=0$ or $r \in a n n M$. Thus $M$ is a prime module.

The following two results are characterizations of SS-coprime modules.

\section{Proposition 2.6:}

Let $M$ be an R-module. Then $M$ is SS-coprime module if and only if for each ideals $I, J$ of $R I J M<<M$ implies $I M=0$ or $J M=0$

\section{Proof :}




\section{ISSN 2347-1921}

$(\Rightarrow)$

Let $\mathrm{I}, \mathrm{J}$ be ideals of $\mathrm{R}$ and $\mathrm{IJM}<<M$. Suppose $\mathrm{JM} \neq(0)$. Hence there exists $b \in J, b \neq 0$ such that $b M \neq 0$. It follows that for each $a \in I, a b M \leq I J M<<M$. So that $a b M<<M$ But $M$ is SS-coprime and $b M \neq(0)$, so that $a M=0$ for each a $\in I$. Thus $I M=(0)$.

$(\Longleftarrow)$ It is clear.

\section{Proposition 2.7 :}

An R-module $M$ is SS-coprime if and only if for each $a, b \in R$, abM $<<M$ implies [aM:M]=annM or [bM:M]=annM.

\section{Proof :}

It is easy so is omitted

\section{Remark 2.8 :}

The homomorphic image of SS-coprime is not necessarily SS-coprime, for example:

The $Z$-module $Z$ is SS-coprime. Let $\pi: Z \rightarrow Z /<6>\simeq Z_{6}$ be the natural epimorphism $\pi(Z)=Z_{6}$ which is not SS-coprime.

\section{Proposition 2.9:}

Let $\mathrm{M}$ be an SS-coprime R-module. Let $\mathrm{N}<<\mathrm{M}$. Then $\frac{M}{N}$ is an SS-coprime R-module.

\section{Proof :}

Let $a, b \in R$ and $a \cdot b\left(\frac{M}{N}\right) \ll<\frac{M}{N}$. Then $\frac{a b M+N}{N} \ll \frac{M}{N}$ and since $N<<M$, we get abM+N $\ll<$, and since $N<<M$, then abM $<<M$. But $\mathrm{M}$ is SS-coprime, so either $\mathrm{aM}=0$ or $\mathrm{bM}=0$. It follows that $\mathrm{a} \frac{\mathrm{M}}{\mathrm{N}}=(0)$ or $\mathrm{b} \frac{\mathrm{M}}{\mathrm{N}}=(0)$. Thus $\frac{M}{N}$ is SS-coprime .

\section{Corollary 2.10 :}

Let $\mathrm{f}: \mathrm{M} \rightarrow \mathrm{M}^{\prime}$ be an epimorphism with kerf $<<M$. If $\mathrm{M}$ is an SS-coprime R-module, then $\mathrm{M}^{\prime}$ is SS-coprime.

\section{Corollary 2.11 :}

Let $\mathrm{M}$ be an R-module with projective cover $\mathrm{f}: \mathrm{P} \rightarrow \mathrm{M}$. If $\mathrm{P}$ is an SS-coprime R-module, then $\mathrm{M}$ is SS-coprime.

\section{Corollary 2.12 :}

Let be a ring. Then the following statements are equivalent

1) Every projective R-module is SS-coprime

2) Every R-module M having a projective cover is SS-coprime .

\section{Proof :}

$(1) \Rightarrow(2)$

It is following directly by Corollary 2.11 .

$(2) \Rightarrow(1)$ Let $M$ be a projective R-module. Since there exists the identity mapping $i: M \rightarrow M$ and kerf $=0<<$, then $M$ has a projective cover. Hence by (2), M is SS-coprime.

\section{Proposition 2.13:}

Let $\mathrm{M}$ be an R-module. Let $\mathrm{N}<\mathrm{M}$ such that $[\mathrm{N}: \mathrm{M}]=\operatorname{annM}$. If $\frac{\mathrm{M}}{\mathrm{N}}$ is an SS-coprime R-module, then $\mathrm{M}$ is SS-coprime.

\section{Proof :}

Let $a, b \in R$ and $a \cdot b M<<M$. It follows that $\frac{a b M+N}{N} \ll \frac{M}{N}$, that is $a b\left(\frac{M}{N}\right) \ll<\frac{M}{N}$. But $\frac{M}{N}$ is $\quad$ SS-coprime, so either $a \frac{M}{N}=(0)$ or $b \frac{M}{N}=(0)$. This implies either $a M \subseteq N$ or $b M \subseteq N$, so either $a \in[N: M]=a n n M$ or $b \in[N: M]=a n n M$. Thus $a M=(0)$ or $b M=(0)$.

\section{Remark 2.14 :}

1) A direct summand of SS-coprime module may not be SS-coprime , for example: consider the $Z$-module $M=Z \oplus Z$. It is easy to see that $M$ is SS-coprime , but by Remark and Example 2.2(1) , $Z_{6}$ is not SS-coprime .

2) The direct sum of SS-coprime modules need not be SS-coprime module , for example: each of the Z-module $Z_{2}$ and $Z_{3}$ is SS-coprime, but $M=Z_{2} \oplus Z_{3} \cong Z_{6}$ is not SS-coprime.

\section{Proposition 2.15 :}


Let $M_{1}$ and $M_{2}$ be $R$-modules and annM $=a n n M_{2}$. Then $M=M_{1} \oplus M_{2}$ is SS-coprime. Particularly, $M \oplus M$ is SS-coprime if $M$ is SS-coprime.

\section{Proof :}

Let $a, b \in R$ and $a b\left(M_{1} \oplus M_{2}\right)<<M_{1} \oplus M_{2}$. Then $a b M_{1}<<M_{1}$ and $a b M_{2}<<M_{2}$. As $M_{1} \& M_{2}$ are SS-coprime, then (either $\mathrm{aM}_{1}=0$ or $\mathrm{bM}_{1}=0$ ) and (either $\mathrm{aM}_{2}=0$ or $\mathrm{bM}_{2}=0$ ). But $\mathrm{annM}_{1}=a \mathrm{nnM}_{2}$, hence $\mathrm{aM}=0$ or $\mathrm{bM}=0$. Thus $\mathrm{M}$ is SS-coprime .

\section{Proposition 2.16 :}

Let $M=M_{1} \oplus M_{2}$. if $M$ is an SS-coprime R-module such that annM $M_{1}$ and annM $M_{2}$ are noncomparable prime ideals, then $M_{1}$ and $\mathrm{M}_{2}$ are SS-coprime modules.

\section{Proof :}

Since $M$ is SS-coprime, then $M$ is S-coprime by Remarks and Examples 2.2(2). Hence by [7, Theorem 19], $M_{1}$ and $M_{2}$ are S-coprime modules. But ann $M_{1}$ and ann $M_{2}$ are prime ideals of $R$, so by Remarks and Examples 2.2(2), $M_{1}$ and $M_{2}$ are SS-coprime modules.

Recall that an R-module $M$ is called small prime if annM=annN for each $(0) \neq N<<M$. Equivalently $M$ is a small prime $R$ module if $(0)$ is a small prime submodule, where a proper submodule $N$ of $M$ is called a small prime submodule if whenever $r \in R, x \in M$ and $(x)<<M$, $r x \in N$ implies $x \in N$ or $r \in[N: M][8]$.

It is clear that every prime module is a small prime module, and if $M$ is a small prime module, then annM is a prime ideal [8]

\section{Theorem 2.17:}

Let $\mathrm{M}$ be an R-module such that every submodule $\mathrm{N}$ of $\mathrm{M}$ is relatively divisible (i.e. $r \mathrm{MnN}=\mathrm{rN}, \forall \mathrm{r} \in \mathrm{R}$ ). If $\mathrm{M}$ is small prime , then $\mathrm{M}$ is SS-coprime.

\section{Proof :}

We claim that $M$ is S-coprime. So I shall prove that annM=[N:M] for each $N<<M$.

Suppose that there is a small submodule $N$ of $M$ and $r \in R, r \neq 0$ such that $r \in[N: M]$ and $r M \neq(0)$. As $M$ is small prime, we get $r N \neq(0)$. By hypothesis, $N$ is relatively divisible, hence $r M n r N=r^{2} N$ and so $r N=r^{2} N$. This implies that, for any $n \in N, r n=r^{2} n_{1}$ for some $n_{1} \in N$, and hence $r\left(n-r n_{1}\right)=0$. But $n-r n_{1} \in N<<M$, so that $\left(n-r n_{1}\right)<<M$. On the other hand, $M$ is small prime, so annM=ann $\left(n-r n_{1}\right)$. Hence $r \in a n n M$, which is a contradiction. Thus annM $=\operatorname{ann}(N: M), \forall N<<M$, i.e. $M$ is $S$-coprime . Beside this , $M$ is small prime implies annM is a prime, so by Remark and Example 2.2(2), $M$ is SS-coprime.

Recall that an R-module $\mathrm{M}$ is called $\mathrm{F}$-regular if $\mathrm{IM \cap N}=\mathrm{IN}$ for each $\mathrm{N} \leq \mathrm{M}$ and each ideal I of $\mathrm{R}$ [5].

\section{Corollary 2.18 :}

Let $\mathrm{M}$ be an F-regular R-module .If $\mathrm{M}$ is small prime, then $\mathrm{M}$ is SS-coprime.

\section{Corollary 2.19 :}

Let $\mathrm{M}$ be amodule over a regular ring $\mathrm{R}$ (i.e. $\mathrm{R}$ is regular in sense of VonNeuamann)

Then the following statements are equivalent :

1) $M$ is a small prime $R$-module

2) $\mathrm{M}$ is an SS-coprime R-module

3) $\mathrm{M}$ is a prime R-module

\section{Proof :}

$(1) \Rightarrow(2)$

Since $R$ is regular ring, $R / a n n(x)$ is a regular ring for each $x \in M$. Hence $M$ is F-regular[14]. Thus the result follows by Corollary 2.18 .

$(2) \Rightarrow(3)$

Since M is SS-coprime, then annM is a prime ideal by Remarks and Examples 2.2(2), so that $\bar{R}=\mathrm{R} / \mathrm{annM}$ is an integral domain. But $\mathrm{R}$ is regular ring implies $\bar{R}$ is regular ring, it is follows that $\bar{R}$ is a field, hence $\mathrm{M}$ is a prime $\bar{R}$-module which implies that $\mathrm{M}$ is a prime R-module .

(3) $\Rightarrow$ (1)

It is clear.

\section{Remark 2.20 :}


Let $\mathrm{M}$ be a divisible module over an integral domain $\mathrm{R}$. Then $\mathrm{M}$ is a faithful SS-coprime.

\section{Proof :}

Let $a, b \in R$ and $a b M<<M$. If $a b \neq 0$, then $a b M=M$, so $M<<M$ which is a contradiction. Thus $a b=0$ and hence $a=0$ or $b=0$. So that $\mathrm{aM}=0$ or $\mathrm{bM}=0$; that is $\mathrm{M}$ is SS-coprime.

Also, if $r \in a n n M$, then $r M=0$. Since $M$ is divisible, then $r=0$. Thus $M$ is faithful.

\section{Proposition 2.21:}

Let $\mathrm{M}$ be a faithful R-module. Consider the following statements :

1) $\mathrm{M}$ is $\mathrm{SS}$-coprime

2) $R$ is SS-coprime

3) $R$ is an integral domain

Then (1) $\Rightarrow(3) \Leftrightarrow(2)$ and (3) $\Rightarrow(1)$ if $\mathrm{M}$ is finitely generated multiplication R-module.

\section{Proof :}

(1) $\Rightarrow(3)$

Let $a, b \in R$ such that $a b=0$. Then $(a b)<<R$. So , $a b M=(0)<<M$. But $M$ is SS-coprime, so either $a M=0$ or $b M=0$. Since $M$ is faithful, then $a=0$ Or $b=0$.

(3) $\Rightarrow(1)$

Let $a, b \in R$ and $a b M<<M$. Since $M$ is finitely generated faithful multiplication module, then $(a b)<<R$. But $R$ is an integral domain , so $(a b)=(0)$ and hence either $a=0$ or $b=0$. Thus either $a M=(0)$ or $b M=(0)$

(3) $\Leftrightarrow(2)$

See Remarks and Examples 2.2(8)

Let $M$ be an R-module, we say that $M$ is small retractable if $\operatorname{Hom}(M, N) \neq 0$ for each $N<<M$.

\section{Proposition 2.22 :}

Let $\mathrm{M}$ be a small retractable and scalar module. If $\mathrm{M}$ is $\mathrm{S}$-coprime, then $\mathrm{RadM}=(0)$.

\section{Proof :}

Suppose there exists $m \in \operatorname{Rad} M, m \neq 0$. Hence $(m)<<M$ and since $M$ is small retractable, there exists $f: M \rightarrow(m), f \neq 0$, hence $f \in$ EndM. But $M$ is a scalar R-module, so that there exists $r \in R$ such that $f(x)=r x, \forall x \in M$. Thus $f(M)=r M \subseteq(m)<<M$ and as $M$ is $S$-coprime, we get $r M=0$. Hence $f=0$ which is a contradiction, therefore $\operatorname{RadM}=(0)$.

Hence it is clear that if $M$ is small retractable scalar module and $M$ is SS-coprime then RadM=0 .

\section{Proposition 2.23 :}

Let $\mathrm{M}$ be an R-module. If $\operatorname{Hom}(M, N)=0$, for each $\mathrm{N}<<M$, then $M$ is S-coprime

\section{Proof :}

Let $a \in R$ and $a M<<M$. Define $f: M \rightarrow M$ by $f(m)=a m, \forall m \in M$. Hence $f(M)=a M<<M$, thus $f \in N o m(M, a M)$ and $a M<<M$, so by hypothesis $f=0$. Thus $f(M)=a M=0$ and $M$ is $S$-coprime.

\section{Corollary 2.24 :}

Let $\mathrm{M}$ be an R-module. If $\mathrm{Hom}(\mathrm{M}, \mathrm{N})=0$ for each $\mathrm{N}<<\mathrm{M}$ and annM is a prime ideal . Then $\mathrm{M}$ is $\mathrm{SS}$-coprime.

\section{Proof :}

It follows by Proposition 2.23 and Remarks and Examples 2.2(2) .

\section{Proposition 2.25 :}

Let $\mathrm{M}$ be an R-module. Then $\mathrm{M}$ is an SS-coprime $\mathrm{E}$-module if and only if $\operatorname{Hom}(\mathrm{M}, \mathrm{N})=0, \forall N<<M$ and ann $\mathrm{E}$ is a prime ideal in $\mathrm{E}$, where $\mathrm{E}=\mathrm{End}(\mathrm{M})$.

\section{Proof :}

$(\Longrightarrow)$ Let $f \in \operatorname{Hom}(M, N), N<<M$. Then $f(M) \subseteq N<<M$, so $f(M)<<M$. But $M$ is SS-coprime E-module, hence $M$ is S-coprime $E$ module and so $f(M)=0$. Thus Hom $(M, N)=0$. Moreover, since $M$ is SS-coprime $E$-module, $a_{E} M$ is a prime ideal in $E$ by Remarks and Examples 2.2(2) . 
$(\Leftarrow)$ First we shall prove $M$ is an S-coprime E-module. Let $f \in \operatorname{Hom}(M, N), f(M)<<M$. Put $f(M)=N$, hence $f \in H o m(M, N)=0$. Thus $\mathrm{f}=0$ and so $\mathrm{M}$ is an S-coprime $\mathrm{E}$-module. But $\operatorname{ann}_{\mathrm{E}} \mathrm{M}$ is a prime ideal so $\mathrm{M}$ is SS-coprime E-module by Remarks and Examples 2.2(2) .

Under the class of multiplication module, we have the following

\section{Theorem 2.26 :}

Let $\mathrm{M}$ be a multiplication R-module. Then $\mathrm{M}$ is an SS-coprime if and only if $\mathrm{M}$ is an SS-coprime E-module .

\section{Proof :}

$(\Rightarrow)$ Let $f, g \in E n d M$, and $(f \circ g)(M)<<M$. Since $g(M) \leq M$ and $M$ is multiplication R-module,

$g(M)=I M$ for some ideal I of $R$. It follows that $(f \circ g)(M)=f(g(M))=f(I M)=\operatorname{If}(M)$. But $f(M) \leq M$, so $f(M)=J M$, for some ideal $J$ of $R$. Thus $(f \circ g)(M)=I J M$ and so $I J M<<M$. But $M$ is an SS-coprime R-module, hence either $I M=0$ or $J M=0$ by Proposition 2.6 . Thus either $f(M)=0$ or $g(M)=0$; that is $M$ is an SS-coprime E-module.

$(\Leftarrow)$ Let $a b M<<M$ where $a, b \in R$. Define $f, g: M \rightarrow M$ by $f(m)=a m, g(m)=b m, \forall m \in M$. Then $(f \circ g)(M)=a b M<<M$. Since $M$ is an SS-coprime E-module, then either $\mathrm{f}(\mathrm{M})=0$ or $\mathrm{g}(\mathrm{M})=0$ and hence either $\mathrm{aM}=0$ or $\mathrm{bM}=0$. Thus $\mathrm{M}$ is an SS-coprime R-module.

Recall that an R-module is called hollow module if every proper submodule of $M$ is small[6].

\section{Proposition 2.27 :}

Let $\mathrm{M}$ be a hollow R-module. Then the following statements are equivalent :

1) $M$ is S-coprime

2) $M$ is coprime

3) $\mathrm{M}$ is SS-coprime

\section{Proof :}

$(1) \Leftrightarrow(2)$ It is clear .

$(1) \Rightarrow(3)$ Let $a b M<<M$ where $a, b \in R$. Then either $a M$ or bM is a proper submodule of $M$. Hence if $a M<M, t h e n a M<<M$ and so $a M=0$. Similarly, $b M=0$. Thus $M$ is SS-coprime.

(3) $\Rightarrow(1)$ It follows by Remarks and Examples 2.2(2).

\section{Proposition 2.28 :}

Let $\mathrm{I}$ be a nil ideal of a ring $R$. If $M$ is an $S$-coprime $R$-module, then $I M=0$.

\section{Proof :}

Let $a \in I$, we claim that $a M<<M$. Assume $a M+K=M$ for some submodule $K$ of $M$. Then for each $n \in Z_{+}, a^{n} M+K=M$. But $a$ is a nilpotent element, so $\mathrm{K}=\mathrm{M}$ and $\mathrm{aM}<<\mathrm{M}$. Since $\mathrm{M}$ is $\mathrm{S}$-coprime, then $\mathrm{aM}=0$ for any $\mathrm{a} \in \mathrm{I}$. Thus $\mathrm{IM}=(0)$.

\section{Proposition 2.29:}

Let $\mathrm{I}$. $\mathrm{J}$ be two ideals of a ring $\mathrm{R}$ such that $\mathrm{IJ}$ is a nil ideal . If $\mathrm{M}$ is an SS-coprime R-module, then $\mathrm{IM}=0$ or $\mathrm{JM}=0$.

\section{Proof :}

Since $\mathrm{M}$ is an SS-coprime R-module, then $\mathrm{M}$ is an S-coprime R-module and hence by Proposition 2.28, IJM=0, so that $\mathrm{IJM}<<\mathrm{M}$. But $\mathrm{M}$ is SS-coprime, therefore either $\mathrm{IM}=0$ or $\mathrm{JM}=0$.

Recall that a ring $R$ is semilocal if $R / J(R)$ is semisimple.

\section{Proposition 2.30:}

Let $R$ be a semilocal ring and $J(R)$ is nilpotent. Then $M$ is S-coprime if and only if $M$ is semisimple.

\section{Proof :}

$(\Rightarrow)$ If $M$ is $S$-coprime. Since $R$ is semilocal,$\frac{R}{J(R)}$ is semilocal, hence $\operatorname{RadM}=J(R) M$ and $\frac{M}{\operatorname{RadM}}$ is semisimple by $[1$. Corollary 15.18]. But $J(R)$ is a nil ideal, so by Proposition 2.28, $J(R) M=0$. then $\operatorname{RadM}=0$.

$(\Longleftarrow)$ It is clear.

\section{Note 2.31:}

If $R$ is a semilocal ring with $J(R)$ is nilpotent and $M$ is an SS-coprime R-module, then $M$ is semisimple, but the converse is not true for example: consider $Z_{6}$ as $Z_{6}$-module. The ring $Z_{6}$ is semilocal, $J\left(Z_{6}\right)=0$ is a nil ideal. Also $Z_{6}$ as $Z_{6}$-module is semisimple, but it is not SS-coprime . 


\section{ISSN 2347-1921}

\section{Semi Strongly S-Coprime Modules}

In this section we investigate the notion of semi strongly S-coprime modules and present some of its properties and some of relations between this concept and other related concepts.

\section{Definition 3.1 :}

An R-module is called semi strongly S-coprime (briefly, SSS-coprime) if for each $a \in R, a^{2} M<<M$ implies $a M=0$.

\section{Remarks and Examples 3.2 :}

1) It is clear that every SS-coprime is SSS-coprime, but not conversely, for example : if $M$ is the $Z$-module $Z_{6}$, then $a^{2} Z_{6}<<Z_{6}$ implies $a^{2} Z_{6}=(0)$; that is $a^{2} \in a n n_{z} Z_{6}=Z_{6}$ and so $a \in 6 Z$. Thus $a Z_{6}=(0)$ and $M$ is SSS-coprime. But $M$ is not SS-coprime.

2) Every SSS-coprime module is S-coprime

\section{Proof :}

Let $M$ be an SSS-coprime module, let $a \in R$ with $a M \ll M$. Since $a^{2} M \subseteq a M$, then $a^{2} M \ll M$. Hence aM=(0) because $M$ is SSS-coprime. Thus M is S-coprime.

3) It is easy to see that : an R-module $M$ is S-coprime and ann $n_{R} M$ is a semiprime ideal of $R$ if and only if $M$ is SSScoprime.

4) Let $M$ be a module over a chained ring $R$. Then $M$ is SS-coprime if and only if $M$ is SSS-coprime .

5) If $M$ and $M^{\prime}$ are isomorphic R-module. Then $M$ is SSS-coprime if and only if $M^{\prime}$ is SSS-coprime.

6) The image of SSS-coprime need not be SSS-coprime. As example to show this : The Z-module Z is SSS-coprime , let $\pi: Z \rightarrow Z /<4>\simeq Z_{4}$ be the natural epimorphism , then $\pi(Z)=Z_{4}$ is not SSS-coprime .

7) For any ring $R \neq 0$. If $R$ is SSS-coprime, then $L(R)=J(R)=(0)$.

\section{Proof :}

Suppose there exists $a \in J(R), a \neq 0$. Then $a^{2} R \ll R$. Since $R$ is SSS-coprime, then $a R=(0)$ (i.e. $\left.a=0\right)$ which is $a$ contradiction. Thus $J(R)=(0)$, hence $L(R)=(0)$.

\section{Proposition 3.3 :}

The direct sum of two SSS-coprime modules is SSS-coprime .

\section{Proof :}

Let $M=M_{1} \oplus M_{2}$, where $M_{1}$ and $M_{2}$ are SSS-coprime R-module. If $r \in R$ such that $r^{2} M \ll M$, then $r^{2} M_{1} \ll M_{1}$ and $r^{2} M_{2} \ll M_{2}$. By SSS-coprimeness of $M_{1}$ and $M_{2}, r M_{1}=(0)$ and $r M_{2}=(0)$. Thus $r M=(0)$ and $M$ is SSS-coprime.

\section{Remark 3.4 :}

A direct summand of SSS-coprime module may be not SSS-coprime, for example : If $M$ is the Z-module $Z \oplus Z_{4}$, then $M$ is SSS-coprime, but $Z_{4}$ is not a SSS-coprime Z-module.

The following result is a characterization of SSS-coprime module .

\section{Proposition 3.3 :}

Let $\mathrm{M}$ be an R-module. Then the following statements are equivalent >

1) $M$ is SSS-coprime module

2) For any ideal $I$ of $R, I^{2} M \ll M$ implies $I M=(0)$

3) For any ideal I of $R$ and $n \in Z_{+}, I^{n} M \ll M$ implies $I M=(0)$.

\section{Proof :}

It is easy, so is omitted.

The following results are analogous to results about SS-coprime modules .

\section{Proposition 3.4 :}

Let $\mathrm{N}<<\mathrm{M}$. If $\mathrm{M}$ is SSS-coprime R-module. Then $\frac{M}{N}$ is an SS-coprime R-module.

\section{Proof :}

It is similar to proof of Proposition 2.9 . 


\section{Corollary 3.7 :}

Let $\mathrm{f}: \mathrm{M} \rightarrow \mathrm{M}^{\prime}$ be an epimorphism with kerf $<<M$. if $\mathrm{M}^{\prime}$ is an SSS-coprime R-module, then $\mathrm{M}$ is SSS-coprime.

\section{Corollary 3.8 :}

Let $\mathrm{M}$ be an R-module with projective cover $\mathrm{f}: \mathrm{P} \rightarrow \mathrm{M}$. If $\mathrm{P}$ is an SSS-coprime coprime.

R-module, then M is SSS-

\section{Proposition 3.9 :}

Let $\mathrm{M}$ be an R-module. Let $\mathrm{N}<\mathrm{M}$ such that $[\mathrm{N}: \mathrm{M}]=\operatorname{annM}$. If $\frac{\mathrm{M}}{\mathrm{N}}$ is an SSS-coprime R-module, then $\mathrm{M}$ is SSS-coprime.

\section{Proof :}

It is similar to the proof of Proposition 2.13

\section{Theorem 3.10 :}

Let $\mathrm{M}$ be a multiplication R-module. Then $\mathrm{M}$ is an SSS-coprime if and only if $\mathrm{M}$ is an SSS-coprime $\mathrm{E}$-module, where $\mathrm{E}=\operatorname{End}(\mathrm{M})$

\section{Proof :}

It is similar to the proof of Proposition 2.26

\section{Remark 3.11 :}

Since every SSS-coprime is S-coprime by Remark and Example 3.2(2). If $R$ is a semilocal ring with $J(R)$ is a nilpotent, then every SSS-coprime is semisimple

Next we have

\section{Proposition 3.12 :}

Let $M$ be a finitely generated faithful multiplication $R$-module. Then M is SSS-coprime if and only if R is SSS-coprime .

\section{Proof :}

$(\Rightarrow)$ Let $\left(a^{2}\right)<<R$. Since $M$ is faithful finitely generated multiplication, then $a^{2} M<<M$, hence $a M=0$. But $M$ is faithful so a=0 (i.e. $(a)=(0))$.

$(\Leftarrow)$ Let $a \in R$ and $a^{2} M<<M$. Since $M$ is faithful finitely generated multiplication, $\left[a^{2} M:{ }_{R} M\right]<<R$, hence $\left(a^{2}\right)<<R$. So $(a)=(0)$. Thus $\mathrm{aM}=(0)$.

\section{References}

[1] F. W. Anderson and K. Fuller, Ring and Categories Springer- Verlag , 1974.

[2] S. Annine, Associated and attached prime over non Commutative Rings , Ph. D. thesis , University of Berkeley (2002)

[3] A. Barnad, Multiplication Modules, J Algebra Vol.71(1981), 174-178

[4] G. Desal, W.K. Nicholson, Endo primitive Rings , J Algebra , Vol 70 (1981), 548-560

[5] D.J. Field house, Pure Theories, Math. Ann. Vol. 184, (1969), 1-18.

[6] P.F. Fleury, Hollow Modules and Local Endo Primitive Rings, Pac. J. Math , Vol 53 (1974), 379-385.

[7] M.A.H. I.M.A.Hadi andR.I.Kahalf, S-coprime Modules, J. of Basrah Researches (Science) Vol. 37 , No. 4. D (2011), 78-86.

[8] L.S. Mahmood, Small Prime Modules and Small Prime submodules, J. of Al-Nahrain, Univ. of Al-Nahrain , Vol. 15 No.4 (2012),191-199.

[9] C.S. Roman, Baer and Quasi Baer Modules, Ph.D. thesis, The Ohio State University , (2004).

[10] S.A. Saymach, On prime submodules, Univ, Tucumare Ser. A. 29 (1979),121-136.

[11] B. N. Shihab , " Scalar Reflexive Modules " , Ph. D. Thesis, University of Baghdad,2004 .

[12] DK, TuTuncu, Tribak R. , On T-nonsingular Modules, Bull. Aust. Math . Soc, 80(2009), 426-471.

[13] S.M. Yassemi, The dual notion of Prime Submodules , Arch. Math. (Bron), Vol. 37,(2001), 273-278.

[14] S.M. Yassen, On F-regular Modules, Ms. C. Thesis . Univ. of Baghdad, 1996.

[15] I. E. Wijayanti, Coprime Modules and Comodules , Ph. D. Thesis , Heirich-Hein Universitat, Dusseldorf, 2006. 